\title{
Photodissociation as a Tool for Nuclear Astrophysics
}

\author{
K. Sonnabend, S. Müller*, M. Elvers, J. Hasper, L. Kern, K. Lindenberg, A. Zilges \\ Institut für Kernphysik, TU Darmstadt, Schlossgartenstraße 9, 64289 Darmstadt, Germany \\ E-mail: kerstin@ikp.tu-darmstadt.de
}

\begin{abstract}
Photodissociation reactions are of crucial interest for the network calculations of stellar nucleosynthesis. On the one hand, they are directly used in $p$-process scenarios changing $s$ - and $r$ process seed distributions to the observed abundance patterns. On the other hand, the study of $(\gamma, n)$ reactions helps to constrain theoretical predictions on the $(n, \gamma)$ cross sections of the branching points on the s-process path. The current status of different experimental efforts is presented and recent developments are discussed.
\end{abstract}

International Symposium on Nuclear Astrophysics - Nuclei in the Cosmos - IX

25-30 June 2006

CERN

* Speaker. 


\section{Introduction}

Three different processes are sufficient to explain the nucleosynthesis of most of the heavy elements: the $s, r$, and $p$ process. The $s$ and $r$ process are based on neutron capture events followed by $\beta$ decays back to the valley of stability. While the $s$ process takes place during stellar burning phases and is characterized by low neutron densities $\left(n_{n} \approx 2-4 \cdot 10^{8} \mathrm{~cm}^{-3}\right)$ and temperatures $\left(T \approx 1-3 \cdot 10^{8} \mathrm{~K}\right)$ [1], the $r$-process is thought to occur in explosive scenarios like, e.g. supernovae and, thus, deals with high neutron densities $\left(n_{n}>10^{20} \mathrm{~cm}^{-3}\right)$ and temperatures $\left(T \approx 1-2 \cdot 10^{9} \mathrm{~K}\right)$ $[2,3]$. The $(n, \gamma)$ cross sections of the so-called branching points on the $s$-process path must be known with good accuracy to predict the isotopic abundance patterns in modern nucleosynthesis models with sufficient correctness. The possibility to constrain the theoretical predictions using photodissociation data is explained in section 2 .

The proton-rich isotopes between $\mathrm{Se}$ and $\mathrm{Hg}$ that cannot be produced by either of these processes are called $p$ nuclei $[4,5]$ and are thought to originate from explosive events lasting a few seconds at temperatures of about $2-3 \cdot 10^{9} \mathrm{~K}$. These $p$ nuclei are produced from $s$ - and $r$-process seed nuclei by photodissociation reactions, like $(\gamma, n),(\gamma, p)$, and $(\gamma, \alpha)$, in a process sometimes referred to as $\gamma$ process [5]. However, the light $p$ nuclei, like e.g. ${ }^{92,94} \mathrm{Mo}$ and ${ }^{96,98} \mathrm{Ru}$, are underproduced in this model framework. The efforts to solve this problem include new nucleosynthesis processes, like e.g. the $r p$ process [6], as well as suggestions of revised cross section data, like e.g. an enhanced ${ }^{22} \mathrm{Ne}(\alpha, n)$ cross section leading to an increase of the $s$-process seed [7]. On that score, the knowledge about photodissociation cross sections plays a crucial role in the prediction of the abundances of the $p$ nuclei. Besides the current status on the experimental efforts, some future projects are presented in section 3 .

\section{Cross Sections of $s$-Process Branching Points}

The neutron capture cross sections of most of the stable isotopes on the $s$-process path have been measured with high accuracy [8]. But the experimental knowledge about the cross sections of the branching points, i.e. unstable isotopes with a decay rate $\lambda_{\beta}$ comparable to the neutron capture rate $\lambda_{n}$, is quite scarce. Recently, the cross sections of some long-living branching points with half-lives in the order of many years were successfully determined using either activation or direct methods (e.g. $\left.{ }^{155} \mathrm{Eu} \mathrm{[9]} \mathrm{and}{ }^{151} \mathrm{Sm}[10,11]\right)$. Severe discrepancies to the theoretically predicted values were found and, therefore, it seems to be favourable to constrain these predictions for the short-living branching points, too.

Most of the short-living branching points play an important role in the final determination of the isotopic abundance patterns due to the fact that the branching is only valid during the high neutron densities provided by the ${ }^{22} \mathrm{Ne}(\alpha, n)$ neutron source in the thermal pulses [12]. However, the preparation of targets enriched in material with half-lives in the order of some dozens of days is nearly impossible. Therefore, data of the inverse $(\gamma, n)$ reaction can be used to observe the same sample of reactants.

Several branching points were investigated at the S-DALINAC using the photoactivation method. After the naturally composed targets were typically irradiated for 24 hours at the nuclear resonance fluorescence setup, the number of the produced unstable isotopes is determined by 
the $\gamma$ rays emitted after their $\beta$ decay. The yield $Y$ of events in the photo-peak is connected to the cross section $\sigma(E)$ and the photon intensity $N_{\gamma}\left(E, E_{\max }\right)$ via

$$
Y \propto N_{\text {target }} \cdot \int_{S_{n}}^{E_{\max }} \sigma(E) \cdot N_{\gamma}\left(E, E_{\max }\right) \mathrm{d} E
$$

with the number of target atoms $N_{\text {target }}$ and the neutron separation energy $S_{n}$. The factor of proportionality consists of the detector efficiency, the $\gamma$ intensity, and some time-dependent factors that are explained in [13] in detail.

Two setups consisting of a HPGe detector and two Low Energy Photon Spectrometers (LEPS), respectively, can be used according to the energy of the emitted $\gamma$ radiation. Results were already published for ${ }^{185} \mathrm{~W}$ [14] and ${ }^{186} \operatorname{Re}[15,16]$ and are in good agreement to direct measurements performed at AIST, Japan [17]. During a recent beam-time, the branching points ${ }^{147} \mathrm{Nd},{ }^{153} \mathrm{Gd}$, and ${ }^{191}$ Os were investigated.

Besides the observation of the short-living branching points, the usage of the inverse $(\gamma, n)$ reaction is also helpful to observe the role and importance of low-lying exited states of the longliving branching points [18]. In the laboratory, only the cross section of the ground-state is normally measured and both, the population numbers and the cross section of excited states remain to be predicted by theory. The excited states are populated in the decay channel of the inverse $(\gamma, n)$ reaction and, thus, the corresponding matrix elements can be studied in this way.

However, the long half-lives and small $\gamma$ intensities that encourage direct cross section measurements are a hindrance for a classical activation experiment due to the fact that the decay of the unstable nuclei cannot be observed. A possible solution is the usage of accelerator mass spectrometry (AMS) for the determination of the yield of unstable nuclei. This method has already been used successfully, e.g. in the case of ${ }^{62} \mathrm{Ni}(n, \gamma)$ and ${ }^{40} \mathrm{Ca}(n, \gamma)$ [19]. Therefore, it is planned to examine some naturally composed targets that were activated during former beam-times with regard to the isotopes ${ }^{93} \mathrm{Zr},{ }^{193} \mathrm{Pt}$, and ${ }^{205} \mathrm{~Pb}$. In a recent beam-time, the reaction ${ }^{152} \mathrm{Sm}(\gamma, n)$ was studied in order to complement the direct measurements of the $(n, \gamma)$ cross section of the branching point ${ }^{151} \mathrm{Sm}[10,11]$.

The combination of activation and AMS enables merely the comparison of all matrix elements involved in the $(\gamma, n)$ reaction to the corresponding theoretical prediction. Therefore, it is planned to study the population of excited states in the daughter nucleus of the $(\gamma, n)$ reaction and the corresponding matrix elements by direct measurements at the NEPTUN tagger system which is under construction at the S-DALINAC.

\section{Reaction Rates for $p$-Process Network Calculations}

Using the photoactivation technique at the S-DALINAC, it is possible to calculate the groundstate reaction rates for $p$-process network calculations from activations at different energies without any assumption about the photodissociation cross section. This method was explained in detail in [13] and results on several isotopes (e.g. ${ }^{190,192,198} \mathrm{Pt},{ }^{197} \mathrm{Au},{ }^{196,198,204} \mathrm{Hg}$, and ${ }^{204} \mathrm{~Pb}$ ) were presented in [20].

Although the sensitivity of the activation technique enables to measure the reaction rates of the very low-abundant proton-rich nuclei in some isotopic chains (e.g. the natural abundance of ${ }^{196} \mathrm{Hg}$ 


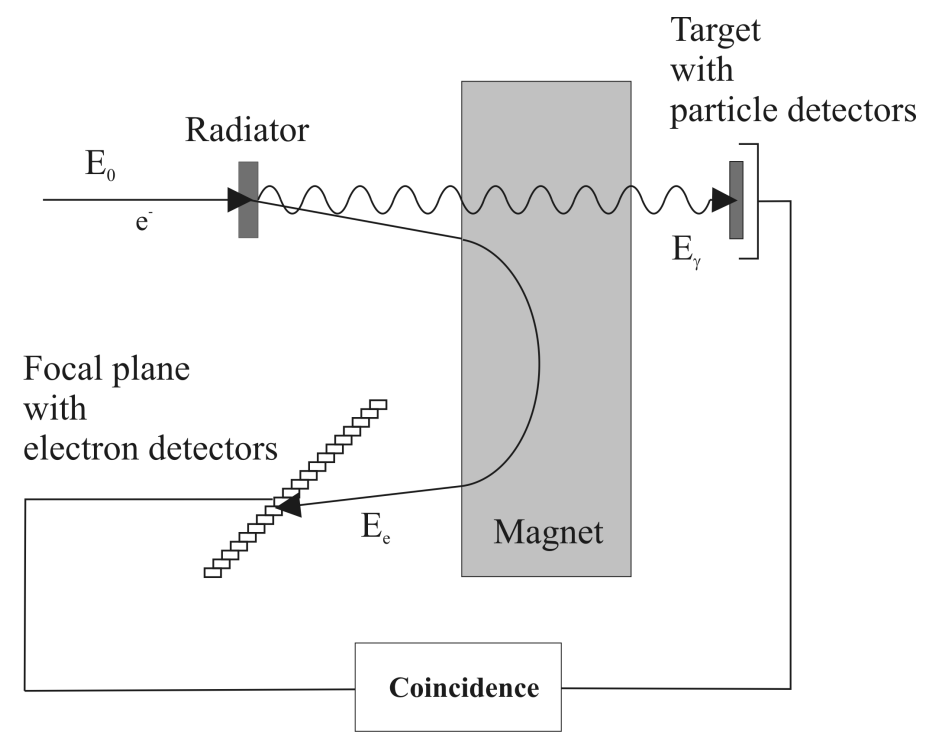

Figure 1: Scheme of the NEPTUN setup.

equals $0.15 \%$ ) unstable isotopes cannot be examined. Therefore, it is planned to use Coulomb Dissociation (CD) in inverse kinematics as it is performed at the SIS/FRS/LAND setup at GSI Darmstadt [21, 22]. The $(\gamma, n)$ cross sections of the isotopes ${ }^{92,93,94,100}$ Mo have been studied in collaboration with the research centers Rossendorf and Karlsruhe. To establish the accuracy of the CD method, the cross sections of ${ }^{92} \mathrm{Mo}(\gamma, n)+(\gamma, p)[23]$ and ${ }^{100} \mathrm{Mo}(\gamma, n)$ were observed at the ELBE accelerator at Rossendorf and at the S-DALINAC at Darmstadt, respectively.

The data of the activation experiments is compared to the results of the CD measurements to estimate the influence of several potential complications on the interpretation of data taken in $\mathrm{CD}$ experiments. The contribution of nuclear break-up and the fraction of different multiplicities to the cross section have to be taken into account as well as the final-state interactions known as post-Coulomb acceleration to extract a reasonable result of the $\mathrm{CD}$ data.

Furthermore, the experimental knowledge about the $(\gamma, p)$ and $(\gamma, \alpha)$ reactions in the Gamow window corresponding to typical $p$-process temperatures is very scarce. Thus, it is planned to use tagged photons at the new setup NEPTUN at the S-DALINAC to fill this gap. The scheme of the tagger is shown in Fig. 1 and the characteristics of the NEPTUN setup are listed in Tab. 1. The NEPTUN setup will be used for the study of $(\gamma, x)$ reactions with $x$ being scattered photons, neutrons, protons or $\alpha$ particles. First test experiments are planned in 2006.

\section{Conclusion and Outlook}

Photodissociation reactions play an important role in nuclear astrophysics. Besides the direct influence of $(\gamma, n),(\gamma, p)$, and $(\gamma, \alpha)$ reactions on the nucleosynthesis in $p$-process scenarios, the study of $(\gamma, n)$ reactions helps to constrain theoretical predictions for the inverse $(n, \gamma)$ cross sections of branching points on the $s$-process path. In the past, the study of photodissociation was realized 


\begin{tabular}{|l|l|}
\hline Radiator target & $\mathrm{Ni}, \mathrm{Al}, \ldots$ \\
\hline Thickness & $\approx 10 \mathrm{mg} / \mathrm{cm}^{2}$ \\
\hline Magnet & $180^{\circ}$ Clam-Shell magnet \\
\hline Focal plane detectors & scintillating fibers (width: $1 \mathrm{~mm}$ ) \\
\hline Number of detectors & 32 (upgrade: 128 ) \\
\hline Energy range & $8 \mathrm{MeV} \leq E_{\gamma} \leq 20 \mathrm{MeV}$ \\
\hline Energy resolution & $\Delta E=25 \mathrm{keV} @ 10 \mathrm{MeV}$ \\
\hline Photon current & $\approx 10^{4} \mathrm{keV}^{-1} \mathrm{~s}^{-1}$ \\
\hline
\end{tabular}

Table 1: Designed characteristics of the NEPTUN setup.

using different photon sources, like e.g. bremsstrahlung or Compton backscattered photons, and different methods, like $e$.g. activation or direct measurements.

New techniques to study the cross sections of unstable isotopes are the usage of Coulomb Dissociation in inverse kinematics and the study of the inverse reactions [24]. Besides the extension of the experimental efforts to unstable isotopes, new methods and setups will be used to increase the accuracy of the "classical" measurements, e.g. the usage of accelerator mass spectroscopy in activation experiments and the availability of tagged photons in a suitable energy range for direct measurements.

\section{Acknowledgments}

We thank T. Rauscher, E. Khan, and S. Goriely for valuable discussions concerning theoretical aspects. F. Käppeler and A. Mengoni are cordially acknowledged for their support in experimental questions. This work was supported by the DFG under contract SFB 634.

\section{References}

[1] F. Käppeler, The Origin of the Heavy Elements: The s Process, Prog. Part. Nucl. Phys. 43 (1999) 419.

[2] K.-L. Kratz, J.-P. Bitouzet, F.-K. Thielemann, P. Möller, B. Pfeiffer, Isotopic r-process abundances and nuclear structure for from stability: implications for the r-process mechanism, Astrophys. J. 403 (1993) 216.

[3] G. Wallerstein, I. Iben, P. Parker, A.M. Boesgaard, G.M. Hale, A.E. Champagne, C.A. Barnes, F. Käppeler, V.V. Smith, R.D. Hoffman, F.X. Timmes, C. Sneden, R.N. Boyd, B.S. Meyer, D.L. Lambert, Synthesis of the elements in stars: forty years of progress, Rev. Mod. Phys. 69 (1997) 995.

[4] D.L. Lambert, The p-nuclei: abundances and origins, Astron. Astroph. Rev. 3 (1992) 3.

[5] M. Arnould, S. Goriely, The p-process of stellar nucleosynthesis: astrophysics and nuclear physics status, Phys. Rep. 384 (2003) 1.

[6] H. Schatz, A. Aprahamian, J. Görres, M. Wiescher, T. Rauscher, J.F. Rembges, F.-K. Thielemann, B. Pfeiffer, P. Möller, K.-L. Kratz, H. Herndl, B.L. Brown, H. Rebel, rp-process nucleosynthesis at extreme temperature and density conditions, Phys. Rev. 294 (1998) 167. 
[7] V. Costa, M. Rayet, R.A. Zappalà, M. Arnould, The synthesis of the light Mo and Ru isotopes: how now, no need for an exotic solution?, Astron. Astroph. 358 (2000) L67.

[8] F. Käppeler, s-Process nucleosynthesis and the interior of Red Giants, Nucl. Phys. A752 (2005) 500c.

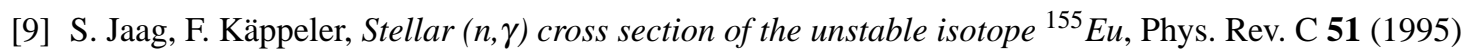
3465.

[10] K. Wisshak, F. Voss, F. Käppeler, M. Krtička, S. Raman, A. Mengoni, R. Gallino, Stellar neutron capture cross section of the unstable s-process branching point ${ }^{151}$ Sm, Phys. Rev. C 73 (2006) 015802.

[11] S. Marrone et al., The n_TOF Collaboration, Measurement of the ${ }^{151} \operatorname{Sm}(n, \gamma)$ cross section from $0.6 \mathrm{eV}$ to $1 \mathrm{MeV}$ via the neutron time-of-flight technique at the CERN n_TOF facility, Phys. Rev. C 73 (2006) 034604.

[12] M. Lugaro, A.M. Davis, R. Gallino, M.J. Pellin, O. Straniero, F. Käppeler, Isotopic compositions of strontium, zirconium, molybdenum, and barium in single presolar SiC grains and asymptotic giant branch stars, Astroph. J. 593 (2003) 486.

[13] K. Vogt, P. Mohr, M. Babilon, J. Endres, T. Hartmann, C. Hutter, T. Rauscher, S. Volz, A. Zilges, Measurement of the $(\gamma, n)$ reaction rates of the nuclides ${ }^{190} \mathrm{Pt},{ }^{192} \mathrm{Pt}$, and ${ }^{198} \mathrm{Pt}$ in the astrophysical $\gamma$ process, Phys. Rev. C 63 (2001) 055802.

[14] K. Sonnabend, P. Mohr, K. Vogt, A. Zilges, A. Mengoni, T. Rauscher, H. Beer, F. Käppeler, R. Gallino, The s-process branching at ${ }^{185}$ W, Astroph. J. 583 (2003) 506.

[15] S. Müller, A. Kretschmer, K. Sonnabend, A. Zilges, D. Galaviz, ${ }^{187} \operatorname{Re}(\gamma, n)$ cross section close to and above the neutron threshold, Phys. Rev. C 73 (2006) 025804.

[16] T. Rauscher, Comment on " ${ }^{187} \operatorname{Re}(\gamma, n)$ cross section close to and above the neutron threshold", Phys. Rev. C 74 (2006) 019801.

[17] T. Shizuma, H. Utsunomiya, P. Mohr, T. Hayakawa, S. Goko, A. Makinaga, H. Akimune, T. Yamagata, M. Ohta, H. Ohgaki, Y.-W. Lui, H. Toyokawa, A. Uritani, S. Goriely, Photodisintegration cross section measurements on ${ }^{186} \mathrm{~W},{ }^{187} \mathrm{Re}$, and ${ }^{188} \mathrm{Os}$ : Implications for the Re-Os cosmochronology, Phys. Rev. C 72 (2005) 025808.

[18] A. Mengoni, Neutron capture cross sections: from theory to experiments and back, Proc. of the Int. Conf. on Nucl. Data for Science and Technology, Santa Fe, NM - September 2004 [nucl-th/0501082]

[19] A. Wallner, R. Golser, W. Kutschera, A. Priller, P. Steier, C. Vockenhuber, AMS - A powerful tool for probing nucleosynthesis via long-lived radionuclides, Eur. Phys. J. A 27 (2006) 337.

[20] K. Sonnabend, K. Vogt, D. Galaviz, S. Müller, A. Zilges, Systematic study of $(\gamma, n)$ reaction rates for $Z \geq 78$ isotopes, Phys. Rev. C 70 (2004) 035802, Erratum: Phys. Rev. C 72 (2005) 019901(E).

[21] T. Aumann, Reactions with fast radioactive beams of neutron-rich nuclei, Eur. Phys. J. A 26 (2005) 441.

[22] K. Sonnabend, M. Babilon, J. Hasper, S. Müller, M. Zarza, A. Zilges, Photodissociation of neutron deficient nuclei, Eur. Phys. J. A 27 (2006) 149.

[23] M. Erhard, A.R. Junghans, R. Beyer, E. Grosse, J. Klug, K. Kosev, C. Nair, N. Nankov, G. Rusev, K.D. Schilling, R. Schwengner, A. Wagner, Photodissociation of p-process nuclei studied by bremsstrahlung-induced activation, Eur. Phys. J. A 27 (2006) 135.

[24] I. Dillmann, M. Heil, F. Käppeler, T. Rauscher, F.-K. Thielemann, Experimental (n, $\gamma$ ) cross sections of the p-process nuclei ${ }^{74}$ Se and ${ }^{84} S r$, Phys. Rev. C 73 (2006) 015803. 